\title{
Estado del arte de la investigación sobre didáctica de la lengua escri- ta en textos argumentativos
}

\section{State of the art of research on didactics of written language in argumentative texts}

(c) UNAN-Managua

Recibido: 24 de febrero 2021

Aprobado: 24 de marzo 2021

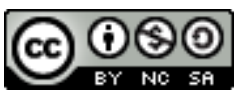

\section{SUMARY}

This article determines its purposes in the research advances of studies related to the didactics of written language in argumentative texts from the cognitive approach, whose methodology corresponds to the phases of documentary research. The first phase, the initial one, determines the problem (didactics of the written language of argumentative texts), ten studies type scientific articles and theses were required. The second phase is the analytical one, with five categories of analysis, the studies at the Latin American level in didactics of the written language and in writing, cognitive approach, the time interval from 2011 to date stand out. The third phase, the final, presents the main findings. In the first category, the common theoretical elements of the investigations belong to didactics of the written language, argumentative written production, cognitive approach. Also, in direct observation techniques, diagnosis, questionnaires, academic illiteracy, and the processes of planning, writing and textual revision. In the second category, Cognitive Approach, there are no studies in Nicaragua, those detected are foreign didactic proposals, some were applied. These emphasize teacher training in written expression, by focusing on writing and reading. Finally, the gaps highlighted in the documentary review are presented, such as the limited study in the textual writing processes to argue, ICT as support tools, academic illiteracy and the lack of didactic proposals with a Cognitive Approach in text studies argumentative to improve written expression.

Keywords: approach, writing, didactics of the written language. las TIC como herramientas de apoyo, el analfabetismo académico y la falta de propuestas didácticas con Enfoque Cognitivo en estudios de textos argumentativos para mejorar la expresión escrita.

Palabras claves: enfoque, redacción, didáctica de la lengua escrita. 


\section{INTRODUCCIÓN}

El presente estudio es una investigación de tipo documental, se estructura a partir de la propuesta de Cifuentes et al., (1993) (como cita Guevara, 2016) al referir tres fases de recopilación y análisis de la información. La primera fase, denominada inicial, referente a identificar el área problemática, en este caso, didáctica de la lengua escrita de textos argumentativos, y la recopilación de la información en diferentes fuentes académicas, se obtuvo diez estudios, los cuales, cinco son a nivel de licenciatura, una en doctora doy cuatro de artículos científicos.

La segunda fase, es denominada analítica, en esta se estableció las cinco categorías de análisis de los documentos. En lo referente a este estudio, en el primero didáctica de la lengua escrita, el segundo estudios latinoamericanos, el tercero enfoque cognitivo, el cuarto estudios en redacción y el quinto un intérvalo temporal de las investigaciones consideradas del año 2011 hasta la época actual.

Desde el punto de vista de Cifuentes et al., (1993) indican que esta fase se realiza la reconstrucción teórica a partir de las relaciones de los argumentos descriptivos en categorías, subcategorías y tendencias (como cita Guevara, 2016). Por consiguiente, como resultado en este estado del arte se establecen como subcategorías las temáticas: didáctica de la lengua escrita, estudios en redacción, enfoque cognitivo, incluyendo la ubicación geográfica (nacional e internacional) de las investigaciones.

La tercera fase es dominada final, en esta según Hoyos (2000) se precisa la interpretación de las categorías y subcategorías temáticas establecidas. En este sentido se proporciona datos investigativos nuevos a través del establecimiento de hipótesis, vacíos, limitaciones, tendencias y logros (como cita Guevara, 2016). Con relación a este estudio, los resultados se presentaron a partir de la comparación y el contraste de las categorías de análisis definidas en la fase dos, desde el enfoque teórico y metodológico, el cual permitió establecer los criterios propuestos por Hoyos (2000). También se diseñó gráficos y tablas para precisar información de las categorías desde una perspectiva geográfica y temporal.

\section{DESARROLLO}

\section{Principales conceptos y teorías Enfoque cognitivo}

Hernández-Morales (2017) argumentan este modelo se basa en las teorías del Enfoque vigotskiano y el Enfoque piagetiano, por tanto, es necesario tener una visión general del modelo cognitivo y su influencia en la enseñanza de redacción de textos argumentativos (como cita Sánchez y Flores, 2020). Del planteamiento anterior de deducen dos posturas. La primera el enfoque cognitivo considera como objeto de estudio la didáctica de la lengua escrita por su amplitud y segundo por su flexibilidad al fusionarse con otros métodos o teorías para desarroIlar procesos mentales elementales que generen ideas en la redacción de textos argumentativos. 
Se debe destacar cita de palabras de Gardner (1987) al exponer que

Un científico que estudia la cognición considera que ésta "debe ser descrita en función de símbolos, esquemas, imágenes, ideas y otras formas de representación mental" es decir, planes, secuencias y estrategias con métodos que desarrollen y ordenen el conocimiento a través de representaciones mentales. (Citado por Hernández 1997, p. 3).

El planteamiento anterior, confirma que el enfoque en mención es una propuesta didáctica utilizada para lengua escrita, además de mejorar en redacción de forma creativa. Quien se comunica coherentemente por escrito, se considera un buen escritor (Cassany et al., 2005).

Los principios que rigen el Enfoque cognitivo propuestos por Rogoff (1984), son cinco, el primero proporciona al alumno un puente entre la información de que dispone (conocimientos previos y nuevos), el segundo una estructura de conjunto para el desarrollo de la actividad o la realización de la tarea, en el tercero se traspasa de forma progresiva el control y la responsabilidad del profesor hacia el alumno, en el cuarto se manifiesta la intervención activa del docente y del alumno y en el quinto aparecen de manera explícita e implícita las formas de interacción habituales entre docentes/alumnos, las cuales no son simétricas, dado el papel que desempeña el profesor como tutor del proceso. (p. 368). En síntesis, todos los principios giran en función de mejorar la didáctica en la lengua escrita y su uso en la redacción de textos desde su análisis, contextos y aspectos del lenguaje escrito.

El Enfoque Cognitivo presenta de manera general tres finalidades según lo expuesto por Caldera (2003).

La primera redefinir el objeto de conocimiento (el lenguaje escrito); la segunda comprender al sujeto que aprende (los alumnos); y la tercera valorar el papel de los docentes como mediadores del proceso de aprendizaje. Se hace evidente de acuerdo a lo expuesto por el autor que este enfoque es activo, tender un puente directo entre los diversos hallazgos de este paradigma y el aula, de tal forma que los docentes estén anuentes a observar cómo es que el alumno aprende, para ascender a nuevas concepciones metodológicas con aportes reflexivos. (p.368)

\section{Competencia comunicativa escrita}

La competencia comunicativa escrita puede considerarse como la habilidad para participar en la sociedad como un comunicador activo y eficiente (Hymes, 1974), lo cual implica en las estrategias de planeación, revisión y corrección de textos a partir de la densidad léxica, precisión gramatical, longitud del enunciado y la ortografía, la adecuación y el contenido, para la competencia sociolingüística; el desarrollo de ideas, la coherencia y la cohesión, para la competencia textual; y el uso de estrategias de planeación y corrección, para la competencia estratégica. (Flores 2014). 
Para hablar como para escribir se requiere interactuar en diversos espacios socioculturales Hasan (1985) confirma esta hipótesis al plantear:

La escritura es, por tanto, producto de un contexto sociocultural que determina sus funciones y requiere de experiencias de redacción propias; se puede caracterizar por situación de distancia espacial y temporal entre quien escribe y quien lee. En concreto la expresión escrita requiere habilidades cognitivas, socioculturales y lingüísticas más sofisticadas que la expresión oral, por lo tanto, la práctica es primordial para mejorar la lengua escrita en lo contextual, actitudinal y procedimental (como cita Flores, 2014, p.46).

De la cita anterior, se puede deducir que la competencia escrita se rige por la práctica y considera los saberes de aprendizajes contextual, procedimental y actitudinal.

La comunicativa escrita consta de tres componentes, estos se relacionan para lograr un mejor aprendizaje en los estudiantes. Desde el punto de vista de Cassany, (1993); Teberosky, (1995); Jolibert, (1997), el primer componente es la planificación o (propósito del escrito, previsible lector, contenido). El segundo es la redacción o textualización (características del tipo de texto, léxico adecuado, morfosintaxis normativa, cohesión, ortografía, signos de puntuación) y el tercer componente es la revisión (el volver sobre lo ya escrito, releyendo y evaluándolo (como cita Caldera, 2003, p. 365).

En la actualidad la retórica juega un papel indispensable para la lengua escrita, junto a paradigmas educativos, que saltan en la búsqueda constante de nuevas concepciones metodológicas que respondan las necesidades de los estudiantes en la época actual. Por tal razón, Flower (1989) propone diversas estrategias para trabajar el proceso global de composición. «Si escribir es un proceso cognitivo complejo, el cual involucra tres subprocesos: planeación, redacción o textualización y revisión» (p. 366), se puede establecer una clasificación de estrategias de la expresión escrita.

Igualmente, los principales problemas que presentan los alumnos en cuanto a redacción de textos reflejados en las últimas investigaciones en Latinoamérica, en el proceso de escritura destacan ideas incoherentes y desorganizadas, sin cohesión ni coherencia, así como, redundancias, vocabulario pobre, usos incorrectos del lenguaje con yeísmo, o queísmos (vicios) por lo cual el tema queda incompleto. Del mismo modo Quispe (2018) expresa que «los estudiantes presentan deficiencias ortográficas, mal uso de los signos de puntuación, escasez de competencia léxica e incoherencias al producir un texto, debido al desconocimiento de los pasos previos a la escritura (¿para qué escribo?, ¿para quién escribo?, ¿sobre qué escribo?»( p. 13).

En relación a la labor del docente se resume en falta y disposición investigativa de estrategias didácticas para mejorar su desempeño educativo. Por consiguiente, es primordial que el docente lidere el interés por la escritura, motivando y guiando a los alumnos a mejorar en redacción de la expresión escrita. 


\section{Trabajos realizados en el área}

El proceso de investigación documental se estructuró a partir de las categorías de análisis mencionado anteriormente en la metodología, de estos, subyacen las subcategorías temáticas: didáctica de la lengua escrita, enfoque cognitivo y estudios en redacción, a manera de resumen la figura 1 se plantea.

Figura 1 Clasificación de las fuentes según categoría temática

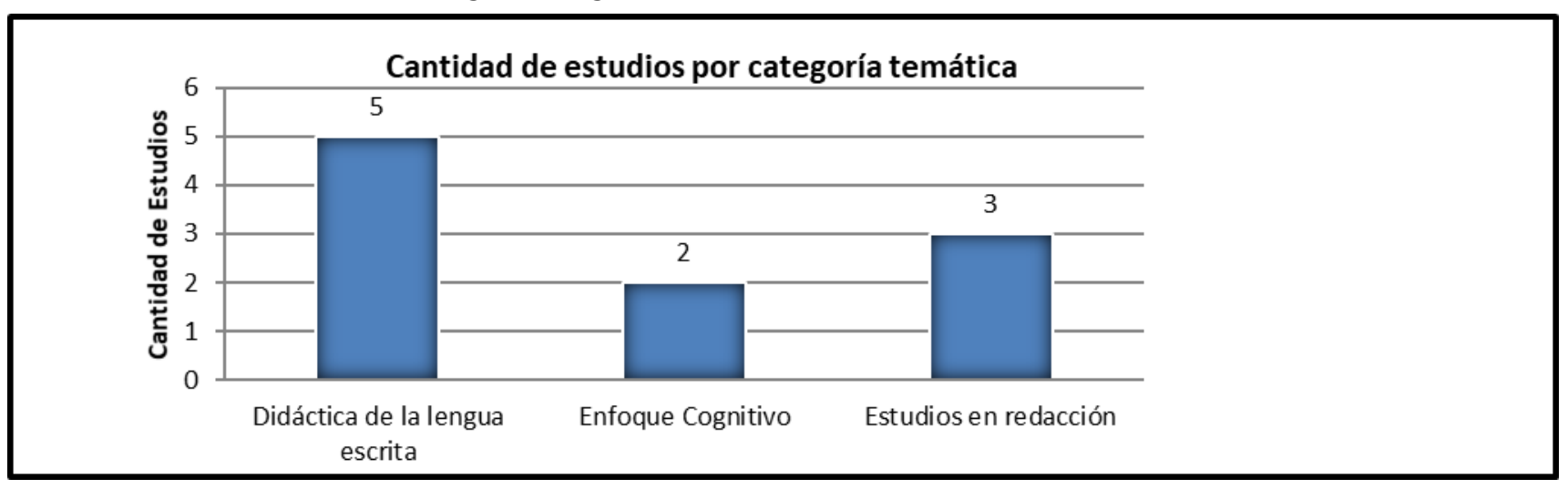

De la figura anterior, se puede deducir las siguientes hipótesis. En primer lugar, de los diez estudios analizados, el 50\% equivalen a la categoría didáctica de la lengua escrita aplicados en los países de Cuba, Perú y Colombia; En segundo lugar, la categoría Enfoque Cognitivo estudiados en los países de Cuba y Colombia a la enseñanza de la lengua escrita corresponden a un 20\% en el análisis bibliográfico, lo que indica que ha sido limitado su estudio en esa habilidad lingüística lamentablemente. Es por esta razón que se proyecta como objetivo bdidáctica de la lengua escrita desde los textos argumentativos.

En tercer lugar, en los países de Chile, Venezuela y Nicaragua se aplicaron estudios que equivalen al 30\% del análisis total de éste trabajo. Es necesario resaltar que de Nicaragua, se destaca un estudio para el desarrollo de la expresión escrita, propuesto por Sánchez y Flores (2020) en su tesis de licenciatura uso de sinónimos para la reescritura de cuentos nicaragüenses con estudiantes de séptimo grado basado en el Modelo de Aprendizaje por Esquemas. Los otros estudios fueron a nivel académico, pero ninguno se aplicó con el enfoque cognitivo de este estudio para resolver el problema delimitado.

Figura 2. Clasificación porcentual según categoría temática

\section{Cantidad de estudios por porcentajes}
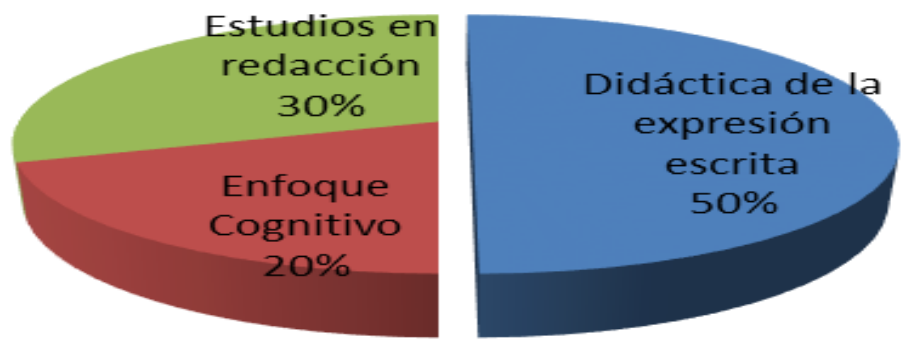


\section{Principales alcances, metodología y resultados de los estudios revisados según las categorías temáticas Di-} dáctica de la lengua escrita

Desde la perspectiva de alcance de los estudios consultados los métodos principales que se aplicaron para dar respuesta a la problemática que se plantea desde la realidad educativa. En mención a las diez investigaciones revisadas, se comprobó que tres son de metodología tipo explicativa cuantitativa. La primera de Valenzuela (2018) es un artículo científico que propone desarrollo y tendencias de las investigaciones relacionadas con la metacognición y los procesos de lectura y escritura académica. La segunda, de Quispe (2018) a nivel de licenciatura aplicó talleres como estrategias didácticas para la redacción de textos argumentativos.

El tercero estudio de Velandia (20011) propone la teoría de Piaget, como explicación del desarrollo cognitivo, y que sirve de base a una teoría del aprendizaje. Luego, se plantea una posible explicación del aprendizaje de campos de conocimiento escolar usando planteamientos piagetianos, pero advierten las limitaciones de este proyecto, por medio de la diferencia entre el estudio básico del aprendizaje y el estudio del aprendizaje en el aula de clases. Estos estudios coinciden en el tipo de Metodología, pero difieren en categorías, uno en redacción de textos, el otro en la didáctica de la lengua escrita a nivel académico, solo el último presenta similitud con el enfoque propuesto en este estudio.

Dentro de este marco de análisis resultó un estudio cualitativo descriptivo, y un cognitivo con metodología comunicativa sociocultural. En relación al primer estudio, es un artículo científico de Montes de Oca (2011), el cual enfatiza el aprendizaje intencional, reflexivo, consciente y autorregulado para utilizar estrategias con métodos adecuados a nivel académico, por deducción teórica. El segundo estudio un artículo científico a nivel de doctorado propuesto por Romeu (2014) el cual sistematiza los aportes teóricos y metodológicos, reflexivos y autocríticos desde la praxis, de la enseñanza de la lengua, asimismo establece la relación coherente entre una teoría del lenguaje y una del aprendizaje, dando explicación al papel del lenguaje en la construcción de sentido del mundo exterior en el proceso de desarrollo cognitivo, afectivo y sociocultural del individuo. Al comparar estas evidencias se puede deducir que ambos estudios son adecuados al nivel académico, con el fin de mejorar la praxis del docente, diferente en cuanto a metodología y enfoque, al igual que en categoría y al problema planteado en este estudio.

Con relación al alcance de metodología aplicada pre experimental con enfoque comunicativo, se comprobó un estudio a nivel de licenciatura propuesto por Manrique (2017), que aplicó la técnica del collage para mejorar la expresión escrita, en técnica, la observación y como instrumento la ficha de observación, apoyado a una secuencia metodológica en capítulos, con resultados eficientes en la textualización de la expresión escrita. Por otro lado Silva (2017) presenta un artículo científico de enfoque comunicativo, para conocer el nivel de redacción de textos académicos, utilizando el método etnográfico, por técnica, la entrevista no estructurada y la observación y el software MAXQDA, como herramienta para organizar y analizar los datos cualitativos, también el estudio de casos como un método de Investigación cualitativa para comprender en detalle y profundi- 
dad la realidad educativa y el ultimo a nivel de artículo científico de enfoque mixto cualitativo y cuantitativo con metodología de investigación documental interpretativa.

En relación a la metodología de las investigaciones mencionadas, la tabla 1 esquematiza los elementos de este criterio:

\section{Tabla 1}

\section{Elementos metodológicos de las investigaciones}

\begin{tabular}{|c|c|c|c|c|c|}
\hline Estudio & Enfoque & Tipo & Técnicas & $\begin{array}{l}\text { Instrumen- } \\
\text { tos }\end{array}$ & $\begin{array}{l}\text { Pro- } \\
\text { puesta } \\
\text { didácti- } \\
\text { ca }\end{array}$ \\
\hline $\begin{array}{l}\text { El estado del arte en la investi- } \\
\text { gación: ¿análisis de los conoci- } \\
\text { mientos acumulados o indaga- } \\
\text { ción por nuevos sentidos? }\end{array}$ & $\begin{array}{l}\text { Cualitativo y } \\
\text { Cuantitativo }\end{array}$ & $\begin{array}{l}\text { Investigación Do- } \\
\text { cumental } \\
\text { interpretativa }\end{array}$ & $\begin{array}{l}\text { Observación } \\
\text { Prueba diagnóstica } \\
\text { Recolección }\end{array}$ & $\begin{array}{l}\text { Cuestiona- } \\
\text { rios } \\
\text { Guía de } \\
\text { observación }\end{array}$ & $\mathrm{SI}$ \\
\hline $\begin{array}{l}\text { La técnica del collage en la ex- } \\
\text { presión escrita de los estudian- } \\
\text { tes }\end{array}$ & Comunicativo & $\begin{array}{l}\text { Aplicada } \\
\text { Pre experimental }\end{array}$ & $\begin{array}{l}\text { Observación directa } \\
\text { Técnica del collage } \\
\text { El registro } \\
\text { El Fichaje }\end{array}$ & $\begin{array}{l}\text { El programa } \\
\text { SPSS } 24 . \\
\text { La ficha de } \\
\text { observación }\end{array}$ & SI \\
\hline $\begin{array}{l}\text { Estrategias docentes y métodos } \\
\text { de enseñanza-aprendizaje en la } \\
\text { Educación Superior }\end{array}$ & Cualitativo & $\begin{array}{l}\text { Descriptivas } \\
\text { interpretativa }\end{array}$ & No se precisa & $\begin{array}{l}\text { No se preci- } \\
\text { sa }\end{array}$ & SI \\
\hline $\begin{array}{l}\text { Aplicación del taller "valoro mis } \\
\text { escritos" utilizando estrategias } \\
\text { didácticas para favorecer la re- } \\
\text { dacción de textos argumentati- } \\
\text { vos en las estudiantes del VII } \\
\text { ciclo de la universidad }\end{array}$ & Cuantitativa & Explicativo & $\begin{array}{l}\text { Software SPSS } \\
\text { Prest test } \\
\text { Post test }\end{array}$ & $\begin{array}{l}\text { Lista de } \\
\text { cotejo } \\
\text { Pruebas de } \\
\text { redacción } \\
\text { Cineforo }\end{array}$ & SI \\
\hline $\begin{array}{l}\text { Aporte de la obra de Piaget a la } \\
\text { comprensión de problemas edu- } \\
\text { cativos: su posible explicación } \\
\text { del aprendizaje }\end{array}$ & Cuantitativo & $\begin{array}{l}\text { Explicativo } \\
\text { Pre experimental }\end{array}$ & $\begin{array}{l}\text { pre prueba- post } \\
\text { prueba }\end{array}$ & $\begin{array}{l}\text { prueba es- } \\
\text { crita } \\
\text { lista de co- } \\
\text { tejo } \\
\text { mapas con- } \\
\text { ceptuales }\end{array}$ & SI \\
\hline $\begin{array}{l}\text { La metacognición en los proce- } \\
\text { sos de lectura y escritura acadé- } \\
\text { mica: ¿qué nos dice la literatu- } \\
\text { ra? }\end{array}$ & $\begin{array}{l}\text { Metacogniti- } \\
\text { vo }\end{array}$ & Explicativo & No se precisa & $\begin{array}{l}\text { No se preci- } \\
\text { sa }\end{array}$ & $\mathrm{SI}$ \\
\hline $\begin{array}{l}\text { Periodización y aportes del enfo- } \\
\text { que cognitivo, comunicativo y } \\
\text { sociocultural de la enseñanza de } \\
\text { la lengua. }\end{array}$ & Cognitivo & $\begin{array}{l}\text { Comunicativo } \\
\text { Funcional }\end{array}$ & Observación & $\begin{array}{l}\text { Encuesta } \\
\text { Aplicada }\end{array}$ & SI \\
\hline $\begin{array}{l}\text { Nivel de redacción de textos } \\
\text { académicos de estudiantes in- } \\
\text { gresantes a la universidad. }\end{array}$ & Comunicativo & Etnográfico & $\begin{array}{l}\text { Entrevistas } \\
\text { La observación par- } \\
\text { ticipante. } \\
\text { - Aplicación del } \\
\text { software MAXQDA, } \\
\text { (herramienta para } \\
\text { organizar y analizar } \\
\text { datos cualitativos }\end{array}$ & $\begin{array}{l}\text { Software } \\
\text { MAXQDA }\end{array}$ & $\mathrm{SI}$ \\
\hline
\end{tabular}


Tomando en cuenta la tabla 1, se deduce lo siguiente: el 80 \% de las investigaciones analizadas utilizan; el enfoque cualitativo un 10\%, el cuantitativo el 20\%, el mixto (cualitativo y cuantitativo) un 10\%, el comunicativo un $20 \%$, un cognitivo el $10 \%$ y un enfoque metacognitivo un10\%, Igualmente con las técnicas, las más aplicadas son la observación, pruebas diagnósticas, entrevistas y encuestas para recopilar y analizar la información, aunque se debe mencionar algunas poco común, como Software SPSS Prest test, Post test, y software MAXQDA a nivel tecnológico. En cuanto a los instrumentos utilizados, el cuestionario, la guía de observación, lista de cotejo, y pruebas de redacción fueron los comunes de todos los estudios.

De las evidencias anteriores se destacan los siguientes elementos comunes de dichas investigaciones. Primeramente, los estudios influyen con el problema planteado (didáctica de la lengua escrita desde los textos argumentativos) de diferentes teorías científicas. Seguidamente, hay propuestas de intervención didáctica para desarrollar la expresión escrita en la redacción de textos académicos y argumentativos, la mayoría se basa en el estudio de planificación, textualización y revisión textual para la redacción de textos. El aporte en estudios de teorías unificadas como estrategias didácticas para mejorar la expresión escrita, a nivel superior y académico.

También Quispe (2016) plantea la metodología del cineforo como herramienta para el desarrollo de habilidades y competencias, además de interpretar analizar, planear y producir textos argumentativos con ajuste a las situaciones y condiciones necesarias, en la escritura. De igual manera, Montes de Oca et al. (2011) sugiere el método aprendizaje basado en problemas, la solución de problemas genera conocimientos y promueve la creatividad, estimula el auto aprendizaje, la argumentación y la toma de decisiones, favorece el desarrollo de habilidades interpersonales y de trabajo en equipo. Asimismo, Sánchez y Flores (2020) proponen que para que el aprendizaje sea efectivo, es necesaria una secuencia didáctica no sólo para el docente en la que diga cómo enseñar, sino en una que se centre en como el estudiante aprende.

Los resultados de las investigaciones analizadas se presentan a partir de dos proposiciones. La primera, relaciona los objetivos que participan en la didáctica propuesta y la segunda observación de la práctica docente. En cuanto al primer punto de vista, la mayoría de investigadores deducen que el planteamiento de propuestas metodológicas debe de estar enfocado en situaciones concreta, en la didáctica de la lengua escritas se debe de analizar el contexto, los propósitos sociales, el modo de organización y los recursos del proceso cognitivo.

De la misma forma el éxito del desarrollo de la expresión escrita radica en integrar elementos que la define como un proceso que involucra al estudiante - docente, desde la praxis, experiencias, conocimientos, capacidad de ordenar ideas, así como estrategias para coordinar múltiples procesos y habilidades, fonológica, morfosintáctica, léxico-semántica y pragmática.

Enrelaciónalaprácticadocenteapartirdelasobservacionesdelosin-

vestigadoresseconcluyóquelamayoríadesconocenlos procesos de planificación, ejecución y evaluación de las acti- 
vidades escritas a nivel áulico. Los recursos didácticos como los libros de textos, diccionarios, pizarra y marcadores. En resumen, la atención está en fortalecer la expresión escrita en todos los aspectos.

\section{Enfoque Cognitivo}

En relación a esta categoría se presentan dos investigaciones con alcance teórico, la primera con base en las teorías del Enfoque vigotskiano y el Enfoque piagetiano propuesta por Hernández y Morales (2017) a nivel de licenciatura. La segunda por Gardner (1987. Al exponer el alcance de la tesis de Hernández y Morales (2017) tipo cualitativo descriptivo, debido a su amplitud y flexibilidad al fusionarse con otros métodos o teorías para desarrollar procesos mentales elementales que generen ideas en la redacción de textos argumentativos. Se fundamenta en la observación de situaciones ya existentes en función de las variables de análisis establecidas, en este caso las actividades y recursos didácticas, técnicas de evaluación y la capacitación docente para el desarrollo dela expresión escrita y textos argumentativos.

Gardner (1987) es de tipo exploratoria, ya que se encargó de examinar el problema de investigación para establecer prioridades de estudio para el futuro, en este caso, el desempeño del docente, en el área de Lengua y Literatura a partir de la aplicación del Enfoque Cognitivo. Además, el planteamiento anterior, confirma que el enfoque en mención es una propuesta didáctica utilizada para lengua escrita, para mejorar la redacción de forma creativa. Quien se comunica coherentemente por escrito, se considera un buen escritor (Cassany et al., 2005).

En relación a la metodología de las investigaciones mencionadas, en la tabla 2 esquematiza los elementos de este criterio:

\section{Tabla 2}

Elementos metodológicos de las investigaciones según Enfoque Cognitivo

\begin{tabular}{|c|c|c|c|c|c|}
\hline \multirow[b]{2}{*}{ Nombre del estudio } & \multicolumn{5}{|c|}{ Elementos Metodológicos } \\
\hline & Enfo- & Tipo & Técnicas & Instrumentos & Propuesta \\
\hline $\begin{array}{l}\text { El Enfoque Cognitivo de la } \\
\text { Escritura y sus Consecuencias } \\
\text { Metodológicas en la Escuela }\end{array}$ & $\begin{array}{l}\text { Cogni- } \\
\text { tivo }\end{array}$ & $\begin{array}{l}\text { Interactivo y } \\
\text { funcional }\end{array}$ & No se precisa & No se precisa & si \\
\hline $\begin{array}{l}\text { Uso de sinónimos para la } \\
\text { reescritura de cuentos nicara- } \\
\text { güenses con estudiantes de } \\
\text { séptimo grado basado en el } \\
\text { Modelo de Aprendizaje por } \\
\text { Esquema }\end{array}$ & $\begin{array}{l}\text { Cuali- } \\
\text { tativo }\end{array}$ & $\begin{array}{l}\text { Descriptivo } \\
\text { IAP }\end{array}$ & $\begin{array}{l}\text { Entrevista, } \\
\text { Encuesta } \\
\text { Prueba } \\
\text { diagnósti- } \\
\text { ca } \\
\text { Observa- } \\
\text { ción }\end{array}$ & $\begin{array}{c}\text { Guía de } \\
\text { Observa- } \\
\text { ción } \\
\text { Videos y gra- } \\
\text { baciones } \\
\text { Cuaderno del } \\
\text { estudiante } \\
\text { Cuestionario }\end{array}$ & si \\
\hline
\end{tabular}


De lo expuesto con anterioridad en la tabla 2, se deduce los siguientes puntos. El primero, se puede estudiar y aplicar el enfoque cognitivo puro o fusionado con aportes metodológicos, y desde un enfoque cualitativo según los objetivos de cada investigador. Se hace evidente que Caldera (2003) toma como objeto de estudio analizar el papel del enfoque cognitivo de la escritura y sus consecuencias metodológicas en la escuela, de la universidad de los Andes, Bogotá, Colombia. Mientras, que Sánchez y Flores (2020) describen dificultades relacionados con la expresión escrita, con medición de variables a través de la estructuración de una secuencia didáctica en la intervención didáctica, en el municipio de Tipitapa, Managua, Nicaragua. En segundo lugar, los instrumentos más utilizados para trabajar este campo fueron la observación, encuesta, entrevista y la secuencia didáctica.

Los resultados de las dos investigaciones analizadas se presentan a partir de los criterios relacionados a las intervenciones didácticas y la práctica docente. En relación al primer criterio, es relevante resaltar que ambos estudios presentan propuestas de intervención didáctica según las dificultades detectadas en la diagnosis de la muestra precisada, uno aplicó y el otro no aplicación. La investigación de Caldera manifiesta en su propuesta elementos para las variables estrategias y actividades didácticas, no se precisa técnica ni instrumentos, solo brinda aportes del paradigma cognoscitivo y aplicaciones a nivel áulico, para el desarrollo de la expresión escrita.

En relación a la primera variable menciona que las actividades didácticas deben de estructurase a partir de las adaptaciones de las necesidades de los estudiantes, estudio del registro lingüístico, paralingüísticos y adaptación al grado de madurez. Igualmente, se sugieren las técnicas de la entrevista, diagnóstica, y la observación, desde los recursos utilizados se deben de crear trabajos grupales, de complete, competencias. Por último, como técnicas evaluativas, recomienda la observación y la evaluación en los diferentes aspectos.

Por otro lado, Sánchez y Flores (2020), pueden ofrecer aportes para mejorar la lengua escrita desde esta categoría. En primer lugar porque ambos estudios se relacionan por fortalecer la competencia de la lengua escrita, y en segundo lugar, porque la propuesta pedagógica de ellas se basa en el aprendizaje por esquemas, el cual pone en práctica todo el sistema del Enfoque cognitivo a plenitud, analizando y fortaleciendo los proceso de acomodación, asimilación y equilibración de los nuevos esquemas de aprendizaje, a través de actividades lúdicas de constante movimiento en los que fortalecerán las demás habilidades (hablar, escuchar, leer). Se puede concluir que, al comparar y contrastar la información de estas variables, es de mucho beneficio para este trabajo de investigación.

\section{Vacíos cognitivos identificados}

El análisis documental de los diez estudios propuestos ayudo a identificar algunos vacíos cognitivos presentes en la mayoría de investigaciones. El primer lugar, los relacionados a la falta de estudios en didáctica de la lengua escrita con mención en redacción en los diferentes métodos educativos, al igual que los estudios en los procesos de redacción textual para argumentar, los cuales son evidentes en las escrituras fragmentadas de los 
estudiantes que tienen dificultades, así como las técnicas para la escritura, por lo tanto, omiten las demás habilidades lingüística, gramatical, ortográfica y el uso de elementos lingüísticos textuales.

El segundo lugar, referente al uso de las TIC para el desarrollo de lasactividadesescritas.Enelanálisisdocumentalsolosetieneconstanciadelestudiode Silva(2017) con Aplicación del software MAXQDA, (herramienta para organizar y analizar datos cualitativos). Asimismo, Sánchez y Flores (2020) al utilizar videos y grabaciones para el modelado de la expresión escrita, de actividad lúdicas, Las TIC deben ser utilizadas como un recurso de apoyo de materias y también para la consecución y progreso de competencias TIC. EL uso de estas no debe ser una acción paralela al proceso de enseñanza, sino que debe estar incorporada, como referente en aplicación móvil en las propuestas didácticas planteadas por los estudios.

El tercer vacío encontrado es el analfabetismo académico de los estudiantes en diferentes niveles educativo, Hall y López (2011) opinan que el término analfabetismo académico es para referirse a las dificultades que presentan los estudiantes que cursan estudios superiores cuando interpretan y producen textos académicos. Referente a las propuestas planteadas por los estudios analizados Valenzuela (2018) lo aborda como ejemplo de problemas en los procesos de lectura y escritura.

El cuarto vacío referente a la falta de propuestas didácticas con Enfoque Cognitivo en estudios de textos argumentativos para mejorarla expresión escrita, se encontró los siguientes vacíos. En primera instancia, cinco propuestas en didáctica de la lengua escrita determinadas en este estudio fueron las de Velandia, Quispe, Manrique, Montes de Oca y Caldera, estas no se aplicaron a la problemática definida, solo fueron sugerencias para enfrentar los hechos delimitados en los instrumentos de investigación de la fase diagnóstica. También, dicho enfoque está orientado a la expresión escrita, no hay estudios en las demás habilidades del lenguaje (oral y la lectura), Caldera, Sánchez y Flores brindan pautas de integración, sin embargo, las habilidades nunca están separadas al momento de activarse. El enfoque cognitivo trabaja a plenitud, analizando y fortaleciendo los procesos de acomodación, asimilación y equilibración de los nuevos esquemas de aprendizaje, a través de actividades de constante movimiento en los que fortalece las demás habilidades (hablar, escuchar, leer).

En ese mismo contexto, el objetivo del Enfoque Cognitivo es desarrollar la competencia escrita, sin embargo, no se confirmó estudios que relacionará todas las habilidades de lengua escrita en una sola propuesta didáctica. En cambio, con otros tipos de competencia desde el Enfoque por Competencias aplicados en países como Nicaragua, el estudio de Sánchez y Flores (2020) nos ofrece aportes didácticos en la expresión escrita y en parte del enfoque cognosci-esquematizado de Jean Piaget (1896-1980) quien desarrolló esa teoría. Otro aspecto importante, es que ninguno de los estudios revisados sugiere el desarrollo de la competencia escrita para estudiantes con problemas de redacción de textos argumentativos a nivel académico, desde el enfoque cognitivo.

Se hace necesario resaltar desde el punto de vista del alcance de investigación, la mayoría de estudios son de tipo exploratorio, hay un mínimo con el enfoque descriptivo y explicativo, al igual que el comunicativos, mix- 
to, etnográfico y metacognitivo Esto indica, que se deben de realizar propuestas didácticas a partir de los alcances no estudiados, lo que permitirá tener una mayor explicación de los fenómenos(problema de investigación) para probar hipótesis y realizar saltos teóricos en busca de concepciones, para ascender a nuevas líneas de investigativas con hipótesis reflexivos y críticos desde la praxis.

\section{CONCLUSIONES}

El dominar las habilidades de lectura y escritura de diversos tipos de textos, es una actividad compleja, pero no imposible. La interrelación de las competencias lingüísticas en el desarrollo de la comunicación escrita es muy amplia, pero necesaria, un buen plan didáctico junto con los aportes de la teoría cognoscitivista a la enseñanza-aprendizaje de la lengua escrita en la escuela permiten redefinir el objeto de conocimiento en el lenguaje escrito, comprender al sujeto que aprende y valorar el papel de los docentes como mediadores del proceso de aprendizaje.

Nos interesa tender un puente directo entre los diversos hallazgos de este paradigma y el aula, de tal forma que los docentes estén en posibilidad de deducir ¿cómo es que el alumno aprende?, con el fin de generar clases más divertidas, interesantes y útiles, para formar niños escritores en la escuela. Investigar es establecer prioridades de estudio para el futuro, en este caso, el desempeño del docente, en el área de Lengua y Literatura a partir de la aplicación del Enfoque Cognitivo. Debido a esto, el planteamiento anterior, confirma que el enfoque en mención es una propuesta didáctica utilizada para lengua escrita, para mejorar la redacción de texto de forma creativa.

Se constató a través de la revisión documental la carencia de investigaciones en didáctica de la lengua escrita. Además, las existentes no precisan un modelo de aprendizaje a seguir que cumpla con todos los criterios propuestos en este estudio, el trabajar con las TIC en las actividades escritas es un problema, ya que no todos los centros educativos gozan de ese beneficio. Desde el punto de vista educativo, es necesario, utilizar cada vez más a nivel áulico herramientas propias de esta generación, para motivar y desarrollar sus habilidades a partir de sus estilos y los modos y formas en que aprenden, ya que cada uno tiene su propio ritmo y modo de aprender.

Pues es necesario crear y aplicar una propuesta didáctica dirigida a mejorar los problemas de redacción de textos de la lengua escrita y mejorar el desempeño del docente apoyado en las TIC en el área de Lengua y Literatura a partir de la aplicación del Enfoque Cognitivo, sin embargo, se recomienda el control de las variaciones, para ser efectiva la propuesta educativa, a través de la observación. 


\section{REFERENCIAS}

Caldera, Reina (2003). El Enfoque Cognitivo de la Escritura y sus Consecuencias Metodológicas en la Escuela. Educere, 6(20),363-368. [fecha de Consulta 25 de Mayo de 2021]. ISSN: 1316-4910. Disponible en: https://www.redalyc.org/articulo.oa?id=35662002

Escamilla 2011 Pág. 128 citado por Largo, M. (2014). Efecto de un programa psicoeducativo en el uso de estrategias de enseñanza de docentes de educación primaria. Maracaibo: Universidad Rafael Urdaneta Vicerectorado académico decanato de postgrado e investigación.

Flores Aguilar, María Dolores. (2014). La competencia comunicativa escrita de los estudiantes de ingeniería y la responsabilidad institucional. Innovación educativa (México, DF), 14(65), 43-60. Recuperado en 05 de junio de 2021, de http://www.scielo.org.mx/scielo.php?script=sci_arttext\&pid=S1665$26732014000200004 \&$ lng $=$ es\&tlng=es.

García, A., \& Ortega, H. (2017). Uso de conectores textuales en la redacción de textos argumentativos en los estudiantes del IPT México-Panamá y el Centro Educativo de Tortí (Doctoral dissertation, Universidad de Panamá).

Guevara,P.

(2016).Elestadodelarteenlainvestigación:Canálisisdelosconocimientosacumuladosoindagaciónpornuevossenti dos?,RevistaFolios.(44),165-179.http://www.scielo.org.co/pdf/folios/n44/n44a11.pdf

Hall, Beatriz, \& López, María Isabel. (2011). Discurso académico: manuales universitarios y prácticas pedagógicas. Literatura y lingüística, (23), 167-192. https://dx.doi.org/10.4067/S0716-58112011000100010

Hernández Salazar, P. (2013). El fenómeno usuarios de la información desde un enfoque cognitivo. Investigación bibliotecológica, 27(61), 107-131

López Sánchez, P. A. (2014). ESTRATEGIAS DIDÁCTICAS PARA LA ENSEÑANZA DE LA ESCRITURA. Revista Torreón Universitario, (6). Recuperado a partir dehttp://www.revistasnicaragua.net.ni/index.php/torreon/article/ view/1104

Manrique Espinoza, N. M., \& Pomacarhua Mendoza, J. (2017). La técnica del collage en la expresión escrita de los estudiantes de la Institución Educativa №36003 Santa Ana Huancavelica, 2016.

Montes de Oca Recio, Nancy, \& Machado Ramírez, Evelio F. (2011). Estrategias docentes y métodos de enseñanza -aprendizaje en la Educación Superior. Humanidades Médicas, 11(3), 475-488. 
Revista Lengua y Literatura ISSN digital 2707-0107 Vol. 7. Núm. 2. julio-diciembre 2021

Recuperado en 11 de abril de 2021, de http://scielo.sld.cu/scielo.php?script=sci_arttext\&pid=S1727$81202011000300005 \&$ lng $=$ es\&tlng $=$ es

Procel, G. (7 de enero de 2015). Estrategias didácticas para corregir la descalcaría en el proceso de aprendizaje en el área de matemática de los niños y niñas de segundo grado del subnivel básica elemental, de la unidad educativa Graciela Atarihuana de cueva, del cantón... Loja: Universidad Nacional de Loja. Obtenido de rediseño de estrategias y técnicas didácticas.

Quispe Alva, M. (2018). Aplicación del taller "valoro mis escritos" utilizando estrategias didácticas para favorecer la redacción de textos argumentativos en las estudiantes del VII ciclo de la universidad el Ángeles de Chimbote, filial Chacas en la provincia Asunción, región Áncash-Perú, año académico 2018.

Sánchez Díaz, M. J., \& Flores López, E. I. (2020). Uso de sinónimos para la reescritura de cuentos nicaragüenses con estudiantes de séptimo grado basado en el Modelo de Aprendizaje por Esquema (Doctoral dissertation, Universidad Nacional Autónoma de Nicaragua, Managua).https://repositorio.unan.edu.ni/13652/1/

Silva, M. V. N., \& Valdez, G. R. L. (2017). Nivel de redacción de textos académicos de estudiantes ingresantes a la universidad. Opción, 33(84), 791-817.

Spychala, M. (2014).El enfoque cognitivo y los modelos de procesamiento de la información. Universidad Adam Mickiewicz, Poznan, Polonia. Polonia. Recuperado de https://cvc.cervantes.es/ensenanza/biblioteca_ele/ asele/pdf/25/25_0923.pdf

VALENZUELA, Ángel. La metacognición en los procesos de lectura y escritura académica: ¿qué nos dice la literatura?. Leng. [online]. 2018, vol.46, n.1, pp.69-93. ISSN 0120-3479. https://doi.org/10.25100/ lenguaje.v46i1.6197

Velandia, N. A., \& Romero, R. F. (2011). Aporte de la obra de Piaget a la comprensión de problemas educativos: su posible explicación del aprendizaje. Revista colombiana de educación, (60), 93-105.

Viñoles, M. A. (2013). Conductismo y constructivismo: modelos pedagógicos con argumentos en la educación comparada. Consejo de Redacción, 2(3), 7. 